\title{
Secondary Metabolites from Asclepias otarioides
}

\author{
Emma Maldonado, ${ }^{1, *}$ Sonia Amador, ${ }^{1}$ and Verónica Juárez-Jaimes ${ }^{2}$
}

${ }^{1}$ Instituto de Química, Universidad Nacional Autónoma de México, Circuito Exterior, Ciudad Universitaria, Coyoacán, 04510, D. F., México.emmaldon@unam.mx

2 Departamento de Botánica, Instituto de Biología, Universidad Nacional Autónoma de México, Apartado Postal 70-367, 04510, D. F., México.

Received October $6^{\text {th }}, 2014$; Accepted November $24^{\text {th }}, 2014$

\begin{abstract}
Chemical study of the aerial parts of Asclepias otarioides led to the isolation of four pentacyclic triterpenes and one cardenolide glycoside. This is the first report on the occurrence of the triterpenes $\mathbf{1}, \mathbf{3}$, and $\mathbf{4}$ in the genus Asclepias.

Key words: Asclepias otarioides; Apocynaceae; Asclepiadoideae; Triterpenes; Cardenolide Glycoside.
\end{abstract}

\section{Introduction}

The American genus Asclepias (fam. Apocynaceae, subfamily Asclepiadoideae) includes about 150 species, 68 of which grow in Mexico, and nearly half of them, are endemic [1-3]. Previous chemical investigations of Asclepias species have shown that different types of steroidal compounds such as cardenolides, pregnanes and androstanes, usually as glycosides, are the main metabolites of these plants [4-6]. However, flavonoid glycosides [7], megastigmane glycosides [8], triterpenes [4, 9], conduritols, and conduritol glycosides [10] have been also isolated from these plants. Asclepias species have ecological significance by their relationship with the monarch butterfly, Danaus plexippus; an insect that sequestrates cardenolides from Asclepias plants as a chemical defense mechanism against predators [11]. Although Asclepias species are considered toxic, some of them are used in folk medicine as anthelmintic, analgesic, cardiotonic, and for the treatment of dermatological problems [3], cancer [4], pleuresy, bronchitis [6], and asthma [9]. This paper describes the isolation and the structure elucidation of the major constituents of the aerial parts of Asclepias otarioides E. Fourn., an herbaceous plant, endemic to Mexico [12].

\section{Results and Discussion}

As result of the chemical study of the aerial parts of $A$. otarioides, three oleanane-type triterpenes (1-3), one lupane-type triterpene (4), one cardenolide glycoside (5), $\beta$-sitosterol glucoside and a mixture of $\beta$-sitosterol/stigmasterol were isolated. The oleanane-type triterpenes were identified as oleanonic acid (1) [13-15], oleanolic acid (2) [16, 17], and 3,4-seco-olean-12-en3,28-dioic acid (3) [18], while the structure of the lupane-type triterpene corresponded to betulinic acid (4) [19, 20] (Fig. 1). Structures of compounds 1-4 were determined by analyses of their IR, MS and NMR spectra and comparison of these data with those reported in the literature. $\beta$-Sitosterol glucoside and
Resumen. El estudio químico de las partes aéreas de Asclepias otarioides condujo al aislamiento de cuatro triterpenos pentacíclicos y de un glicósido de cardenólida. Este es el primer informe sobre la presencia de los triterpenos 1, 3 y $\mathbf{4}$ en el género Asclepias.

Palabras clave: Asclepias otarioides; Apocynaceae; Asclepiadoideae; triterpenos; glicósido de cardenólida.

the mixture of $\beta$-sitosterol/stigmasterol were identified by comparison of their ${ }^{1} \mathrm{H}$ NMR spectra and physical constants with those of authentic samples.

Compound $\mathbf{5}$ was part of a complex mixture from which it could not be isolated. So, the mixture was esterified $\left(\mathrm{Ac}_{2} \mathrm{O}-\mathrm{pyr}-\right.$ idine) and compound $\mathbf{5}$ was isolated as the pentaacetyl derivative 6. The molecular formula of this derivative was assigned as $\mathrm{C}_{39} \mathrm{H}_{54} \mathrm{O}_{15}$ by the pseudomolecular ion at $m / z 785.3356[\mathrm{M}$ $+\mathrm{Na}]^{+}$observed in its HRESIMS (calcd. for, 785.3355). The ${ }^{13} \mathrm{C}$ NMR spectrum of compound 6 showed 39 signals; 10 of them correspond to 5 acetyl groups, another 6 signals were assigned to a monosaccharide, and the remaining 23 signals were atributted to a cardenolide. The ${ }^{1} \mathrm{H}$ NMR spectrum showed the signals for the sugar moiety at $\delta 4.84$ (d, H-1'), 4.79 (dd, H2'), 5.60 (t, H-3'), 4.66 (dd, H-4'), 3.94 (dq, H-5'), and 1.21 (d, H-6'). The coupling constants of these signals $\left(J_{1-2}=8.0\right.$ $\mathrm{Hz}, J_{2-3}=J_{3-4}=3.0 \mathrm{~Hz}, J_{4-5}=10.0 \mathrm{~Hz}$ ) led to the identification of this sugar as $\beta$-allomethylose [21]. The presence of an $\alpha, \beta$-unsaturated- $\gamma$-lactone in the aglycone was deduced from ${ }^{1} \mathrm{H}$ NMR signal at $\delta 5.85$ for the vinyl proton $\mathrm{H}-22$ and those
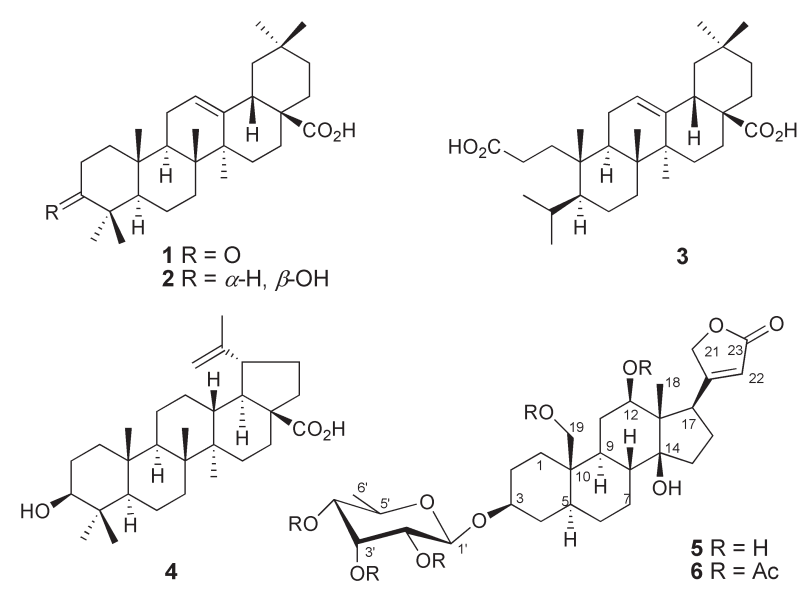

Fig. 1. Structures of compounds 1-6. 
for the $\gamma$-methylene protons $\left(\mathrm{H}_{2}-21\right)$ at $\delta 4.85$ and 4.77; and confirmed by the ${ }^{13} \mathrm{C}$ NMR signals at $\delta 173.0$ (C-20), 73.2 $\left(\mathrm{CH}_{2}-21\right), 118.1(\mathrm{CH}-22)$, and $174.1(\mathrm{C}-23)$. The aglycone also presents signals for one oxymethylene $\left(\delta_{\mathrm{H}} 4.32 \mathrm{~d}, J=12.5 \mathrm{~Hz}\right.$, $\left.\delta_{\mathrm{H}} 4.12 \mathrm{~d}, J=12.5 \mathrm{~Hz} ; \delta_{\mathrm{C}} 61.7, \mathrm{CH}_{2}-19\right)$, two oxymethines $\left(\delta_{\mathrm{H}} 4.53 \mathrm{dd}, J=12.0,4.0 \mathrm{~Hz}, \delta_{\mathrm{C}} 77.1, \mathrm{CH}-12 ; \delta_{\mathrm{H}} 3.65 \mathrm{tt}, J=\right.$ $\left.11.0,5.0 \mathrm{~Hz} ; \delta_{\mathrm{C}} 77.6, \mathrm{CH}-3\right)$, a non protonated carbon bonded to oxygen $\left(\delta_{\mathrm{C}} 85.6, \mathrm{C}-14\right)$, and one methyl group $\left(\delta_{\mathrm{H}} 0.88 \mathrm{~s}\right.$; $\left.\delta_{\mathrm{C}} 10.4, \mathrm{CH}_{3}-18\right)$. These assignments were based on analysis of the 2D NMR spectra, establishing the structure of the cardenolide as that of $12 \beta$-hydroxycoroglaucigenine. The HMBC correlation of $\mathrm{H}-1$ ' to $\mathrm{C}-3$ showed that the allomethylose was bonded to the oxygen at C-3. In the same manner was established that the acetyl groups were bonded to the oxygens at C-12, C-19, C-2', C-3', C-4' and C-6'. The derivative 6 was identified as $12-O, 19-O, 2^{\prime}-O, 3^{\prime}-O, 4^{\prime}-O, 6^{\prime}-O$-pentaacetyl- $12 \beta$ hydroxycoroglaucigenine-3- $O-\beta$-D-allomethyloside, which has not been described previously. Thus, the cardenolide present in $A$. otariodes was identified as $12 \beta$-hydroxycoroglaucigenine-3$O-\beta$-D-allomethyloside (5) [21].

To our knowledge, this is the first report on the ocurrence of oleanonic acid (1), 3,4-seco-olean-12-en-3,28-dioic acid (3), and betulinic acid (4) in Asclepias genus. Oleanolic acid (2) and other pentacyclic triterpenes have been isolated from $A$. syriaca, A. linaria and $A$. speciosa $[9,22,23]$. 12 $\beta$-Hydroxycoroglaucigenin-3-O- $\beta$-D-allomethyloside (5) and its aglycon were isolated from $A$. curassavica [21,24]. Thus, the chemical composition found in A. otarioides was consistent with those found in another species of Asclepias genus, in which the same type of compounds were present. This indicates that not only cardiac glycosides, but pentacyclic triterpenes are relevant constituents of Asclepias species.

\section{Experimental}

General experimental procedures. Melting points (uncorrected) were determined on a Fisher Jones melting point apparatus. Optical rotations were measured on a Perkin Elmer 343 polarimeter. The IR spectra were recorded on a FTIR-Magna 750 spectrophotometer. NMR spectra were recorded on a Varian Unity Plus 500 or on Varian XR-300 spectrometers, using TMS as internal standard. EIMS were measured on a JEOL JMS-AX505HA mass spectrometer. HRESIMS was recorded on a Bruker microTOF II ESI mass spectrometer. Column chromatographies operated with vacuum (CC) were performed on silica gel 60 (Merck G). Thin layer chromatographies (TLC) were carried out on precoated Macherey-Nagel Sil G/UV 254 plates with thicknesses of $0.25 \mathrm{~mm}$.

Vegetal material. The aerial parts of Asclepias otarioides E. Fourn. were collected in the Ajusco Mountain, Southwest Mexico City, in July 2006. V. Juárez-Jaimes authenticated the vegetal material. A voucher specimen (MEXU 1248 428) was deposited at the National Herbarium.

Extraction and isolation. Fresh aerial parts of A. otarioides $(446 \mathrm{~g})$ were extracted with $\mathrm{MeOH}$ and then with EtOAc.
Both extracts were combined $(148.7 \mathrm{~g})$ and fractioned by partition between EtOAc- $\mathrm{H}_{2} \mathrm{O}$ and $\mathrm{BuOH}-\mathrm{H}_{2} \mathrm{O}$, to obtain 44.7 and $8.1 \mathrm{~g}$ of extract, respectively. The EtOAc-soluble extract was fractioned by silica gel column chromatography (CC) with hexane-EtOAc mixtures to obtain five combined fractions (1A to $5 \mathrm{~A}$ ). Purification of fraction $2 \mathrm{~A}$ (eluted with hexane-EtOAc 19:1) by silica gel CC eluted with hexane-EtOAc 19:1 and 9:1 gave fractions 1B-5B. A mixture of the ubiquitous $\beta$-sitosterol/ stigmasterol $(299 \mathrm{mg}$ ) was obtained from fraction 2B. Crystallization of fraction $3 \mathrm{~B}$ led to the isolation of oleanonic acid (1) $\left(\mathrm{mp} 167-169^{\circ} \mathrm{C},[\alpha]_{\mathrm{D}} 93.5, c\right.$ 0.23, $\mathrm{CHCl}_{3}$, lit.: $\mathrm{mp} 170-176$ ${ }^{\circ} \mathrm{C},[\alpha]_{\mathrm{D}}$ 96.6, $c$ 0.23, $\mathrm{MeOH}[13],[\alpha]_{\mathrm{D}} 73.6, c 0.26, \mathrm{CHCl}_{3}$ [15]) Mother liquors of $\mathbf{1}$ were combined with fraction $4 \mathrm{~B}$ and subjected to silica gel $\mathrm{CC}$ eluted with $\mathrm{CH}_{2} \mathrm{Cl}_{2}$, followed by a second $\mathrm{CC}$ eluted with hexane- $i \mathrm{PrOH}$ 98:2 and crystallization to obtain $41.2 \mathrm{mg}$ of betulinic acid (4) $\left(\mathrm{mp} 285-287^{\circ} \mathrm{C},[\alpha]_{\mathrm{D}}\right.$ 7.0, c 0.20, $\mathrm{CHCl}_{3}$, lit.: $\mathrm{mp} 290-292^{\circ} \mathrm{C},[\alpha]_{\mathrm{D}} 7.5, c 0.5$, pyridine $\left.[19],[\alpha]_{\mathrm{D}} 8.0, c 0.37, \mathrm{CHCl}_{3}[20]\right)$, together with an additional amount of 1, to make a total of $3.87 \mathrm{~g}$. Fractions $3 \mathrm{~A}$ (eluted with hexane-EtOAc 17:3 to 7:3) and 5B were combined, decoloured with activated charcoal and subjected to $\mathrm{CC}$ eluted with hexane-EtOAc gradient. Fractions eluted with hexane-EtOAc 9:1 afforded $568 \mathrm{mg}$ of oleanolic acid (2) $\left(\mathrm{mp} 297-299^{\circ} \mathrm{C},[\alpha]_{\mathrm{D}}\right.$

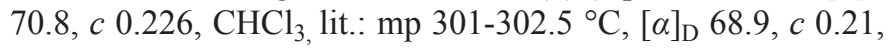
$\left.\mathrm{CHCl}_{3}[16,17]\right)$. Chromatography of fraction 5A (eluted with hexane-EtOAc 1:4 to 0:1) over a silica gel column eluted with $\mathrm{CHCl}_{3}-\mathrm{MeOH}$ 19:1 to17:3 gave fractions 1C-4C. Fraction 1C was purified by $\mathrm{CC}$ eluted with mixtures of $\mathrm{CHCl}_{3}-\mathrm{MeOH}$ of increasing polarity. Fractions eluted with $\mathrm{CHCl}_{3}-\mathrm{MeOH}$ 98:2 gave $21 \mathrm{mg}$ of 3,4-seco-olean-12-en-3,28-dioic acid (3) (mp $268-270{ }^{\circ} \mathrm{C},[\alpha]_{\mathrm{D}} 60.0, c 0.21, \mathrm{CHCl}_{3}$, lit.: $\mathrm{mp}>250{ }^{\circ} \mathrm{C},[\alpha]_{\mathrm{D}}$ 54.4, c 0.006, $\mathrm{MeOH}$ [18]. Crystallization of fraction $2 \mathrm{C}$ gave $53.2 \mathrm{mg}$ of $\beta$-sitosterol glucoside.

Silica gel $\mathrm{CC}\left(\mathrm{CHCl}_{3}-\mathrm{MeOH}\right)$ of the $\mathrm{BuOH}$-soluble extract gave fractions 1D-4D. Repeated CC of fraction 2D gave a mixture $(77 \mathrm{mg}$ ) containing $12 \beta$-hydroxycoroglaucigenin-3-O- $\beta$ $D$-allomethyloside (5), which could not be purified. A portion $(64.4 \mathrm{mg}$ ) of this mixture was acetylated in the usual manner (pyridine $/ \mathrm{Ac}_{2} \mathrm{O}$, room temp., $24 \mathrm{~h}$ ), and purified by silica gel $\mathrm{CC}$ eluted with hexane- $\mathrm{Me}_{2} \mathrm{CO} 4: 1$, to obtain the pentaacetyl derivative of $\mathbf{5}(\mathbf{6}, 71.7 \mathrm{mg})$.

Pentaacetyl-12 $\beta$-hydroxycoroglaucigenin-3-O- $\beta$-D-allomethyloside (6). Amorphous solid; $[\alpha]_{\mathrm{D}}{ }^{20}+5.37\left(c\right.$ 0.28, $\left.\mathrm{CHCl}_{3}\right)$; IR $\left(\mathrm{CHCl}_{3}, v, \mathrm{~cm}^{-1}\right): 1747,1629,1373,1171,1083,1037 ;{ }^{1} \mathrm{H}$ NMR (500 MHz, $\mathrm{CDCl}_{3}, \delta$, ppm, $\left.J / \mathrm{Hz}\right): 2.18(1 \mathrm{H}, \mathrm{dt}, J=14.0$, $3.5 \mathrm{~Hz}, \mathrm{H}-1 \mathrm{a}), 0.91(1 \mathrm{H}, \mathrm{td}, J=14.0,3.5 \mathrm{~Hz}, \mathrm{H}-1 \mathrm{~b}), 1.93(1 \mathrm{H}$, m, H-2a), $1.38(1 \mathrm{H}, \mathrm{m}, \mathrm{H}-2 \mathrm{~b}), 3.65(1 \mathrm{H}, \mathrm{tt}, J=11.0,5.0 \mathrm{~Hz}, \mathrm{H}-$ 3), $1.92(1 \mathrm{H}, \mathrm{m}, \mathrm{H}-4 \mathrm{a}), 1.76(1 \mathrm{H}, \mathrm{m}, \mathrm{H}-4 \mathrm{~b}), 1.25(1 \mathrm{H}, \mathrm{m}, \mathrm{H}-5)$, $1.43(1 \mathrm{H}, \mathrm{m}, \mathrm{H}-6 \mathrm{a}), 1.24(1 \mathrm{H}, \mathrm{m}, \mathrm{H}-6 \mathrm{~b}), 2.02(1 \mathrm{H}, \mathrm{m}, \mathrm{H}-7 \mathrm{a})$, $1.13(1 \mathrm{H}, \mathrm{m}, \mathrm{H}-7 \mathrm{~b}), 1.67(1 \mathrm{H}, \mathrm{td}, J=12.0,3.5 \mathrm{~Hz}, \mathrm{H}-8), 1.08$ $(1 \mathrm{H}, \mathrm{td}, J=12.0,3.0 \mathrm{~Hz}, \mathrm{H}-9), 1.87(1 \mathrm{H}, \mathrm{m}, \mathrm{H}-11 \mathrm{a}), 1.33(1 \mathrm{H}$, m, H-11b), 4.53 (1H, dd, $J=12.0,4.0 \mathrm{~Hz}, \mathrm{H}-12), 1.73(1 \mathrm{H}, \mathrm{m}$, H-15a), 1.36 (1H, m, H-15b), 2.15 (1H, m, H-16a), $1.92(1 \mathrm{H}$, m, H-16b), 2.87 (1H, td, $J=6.0,4.0 \mathrm{~Hz}, \mathrm{H}-17), 0.88(3 \mathrm{H}, \mathrm{s}$, H-18), 4.32 (1H, d, $J=12.5 \mathrm{~Hz}, \mathrm{H}-19 \mathrm{a}), 4.12(1 \mathrm{H}, \mathrm{d}, J=12.5$ Hz, H-19b), 4.85 (1H, br dd, $J=18.0,1.5$ Hz, H-21a), 4.77 (1H, 
dd, $J=18.0,2.0 \mathrm{~Hz}, \mathrm{H}-21 \mathrm{~b}), 5.85$ (1H, br s, H-22); 4.84 (1H, $\mathrm{d}, J=8.0, \mathrm{~Hz}, \mathrm{H}-1^{\prime}$ '), 4.79 (1H, dd, $J=8.0,3.0 \mathrm{~Hz}, \mathrm{H}-2$ '), 5.60 $\left(1 \mathrm{H}, \mathrm{t}, J=3.0, \mathrm{~Hz}, \mathrm{H}-3^{\prime}\right), 4.66$ (1H, dd, $\left.J=10.0,3.0 \mathrm{~Hz}, \mathrm{H}-4^{\prime}\right)$, $3.94\left(1 \mathrm{H}, \mathrm{dq}, J=10.0,6.5 \mathrm{~Hz}, \mathrm{H}-5^{\prime}\right), 1.21(3 \mathrm{H}, \mathrm{d}, J=6.5, \mathrm{~Hz}$, H-6'), 2.15, 2.10, 2.05, 2.02, and 2.01 (3H each, s, $\mathrm{CH}_{3} \mathrm{CO}$ ); ${ }^{13} \mathrm{C}$ NMR (125 MHz, $\left.\mathrm{CDCl}_{3}, \delta, \mathrm{ppm}\right): 31.9(\mathrm{C}-1), 29.2(\mathrm{C}-2)$, 77.6 (C-3), 32.9(C-4), 44.6 (C-5'), 27.9 (C-6), 27.4 (C-7), 41.7 (C-8), 45.9 (C-9), 38.1 (C-10), 27.4 (C-11), 77.1 (C-12), 53.9 (C-13), 85.6 (C-14), 34.4 (C-15), 27.1 (C-16), 45.9 (C-17), 10.4 (C-18), 61.7 (C-19), 173.0 (C-20), 73.2 (C-21), 118.1 (C22), 174.1 (C-23), 96.9 (C-1'), 69.5 (C-2'), 68.8 (C-3'), 71.4 (C-4'), 68.1 (C-5'), 17.5 (C-6'), 21.2, 21.1, 20.74, 20.68, and $20.6\left(\mathrm{CH}_{3} \mathrm{CO}\right), 170.9170 .8,169.8,169.3$, and $169.0\left(\mathrm{CH}_{3} \mathrm{CO}\right)$; FABMS $m / z 763[\mathrm{M}+\mathrm{H}]^{+}(3), 703$ (1), 473 (2), 413 (2), 353 (8), 335 (8), 273 [triacetylallomethylose] ${ }^{+}$(87), 231 (4), 213 (4), 153 (38), 111 (42), 69 (73), 57 (100), 43 (72). HRESI MS $m / z$ 785.3356 [M + Na ${ }^{+}\left(\right.$calcd. for $\left.\mathrm{C}_{39} \mathrm{H}_{54} \mathrm{NaO}_{15}, 785.3355\right)$.

\section{Acknowledgments}

We are very grateful to R. Patiño, H. Ríos, I. Chávez, B. Quiroz, E. Huerta, R. Gaviño, M. A. Peña, N. Zavala, L. Triana, L. Velasco, and J. Pérez for technical assistance.

\section{References}

1. Endress, M. E.; Bruyns, P. V. Bot. Rev. (Lancaster) 2000, 66, 156.

2. Juárez-Jaimes, V.; Alvarado-Cárdenas, L. O.; Villaseñor, J. L. Rev. Mex. Biodivers. 2007, 78, 459-482.

3. Fernández Brewer, A. M.; Juárez Jaimes, V.; Cortés Zárraga, L. Polibotánica 2008, 25, 155-171.

4. Roy, M. C.; Chang, F. R.; Huang, H. C.; Chiang, M. Y. N.;Wu, Y. C. J. Nat. Prod. 2005, 68, 1494-1499.
5. Zhang, R. R.; Tian, H. Y.; Tan, Y. F.; Chung, T. Y.; Sun, X. H.; Xia, X.; Ye, W. C.; Middleton, D. A.; Fedosova, N.; Esmann, M.;Tzen, J. T. C.; Jiang, R.W. Org. Biomol. Chem. 2014, 12, 8919-8929.

6. Warashina, T.; Noro, T. Chem. Pharm. Bull. 2010, 58, 172-179.

7. Haribal, M.; Renwick, J. A. A. Phytochemistry 1996, 41, 139144.

8. Abe, F.; Yamauchi, T. Chem. Pharm. Bull. 2000, 48, 19081911.

9. Araya, J. J.; Kindscher, K.; Timmermann, B. N. J. Nat. Prod. 2012, 75, 400-407.

10. Abe, F.; Yamauchi, T.; Honda, K.; Hayashi, N. Chem. Pharm. Bull. 2000, 48, 1090-1992.

11. Trigo, J. R. J. Braz. Chem. Soc. 2000, 11, 551-561.

12. Juárez-Jaimes, V.; Lozada, L. "Asclepiadaceae" Flora del Valle de Tehuacán-Cuicatlán. Instituto de Biología. Universidad Nacional Autónoma de México. 2003, 37, 1-57.

13. Shirane, N.; Hashimoto,Y.; Ueda, K.; Takenaka, H.; Katoh, K. Phytochemistry 1996, 43, 99-104.

14. Sun, H.; Fang, W. S.; Hu, C. J. Asian Nat. Prod. Res. 2008, 10, 271-276.

15. Ma, C. M.; Nakamura, N.; Hattori, M. Chem. Pharm. Bull. 2000, 48, 1681-1688.

16. Seebacher, W.; Simic, N.; Weis, R.; Saf, R.; Kunert, O. Magn. Reson. Chem. 2003, 41, 636-638.

17. Fukuda, Y.; Sakai, K.; Matsunaga, S.; Tokuda, H.; Tanaka, R. Cancer Letters 2006, 240, 94-101.

18. Caldwell, C. G.; Franzblau, S. G.; Suarez, E.; Timmermann, B. N. J. Nat. Prod. 2000, 63, 1611-1614.

19. Peng, C.; Bodenhausen, G.; Qiu, S.; Fong, H. H. S.; Farnsworth, N. R.; Yuan, S.; Zheng, C. Magn. Reson. Chem. 1998, 36, 267278.

20. Urban, M.; Sarek, J.; Klinot, J.; Korinkova, G.; Hajduch, M. J. Nat. Prod. 2004, 67, 1100-1105.

21. Abe, F.; Mōri, Y.; Yamauchi, T. Chem. Pharm. Bull. 1992, 40, 2917-2920.

22. Adams, R. P.; Tomb, A. S.; Price, S. C. Biochem. Syst. Ecol. 1987, 15, 395-399.

23. Abbott, T. P.; Peterson, R. E.; Tjarks, L. W.; Palmer, D. M.; Bagby, M. O. Econ. Bot. 1990, 44, 278-284.

24. Abe, F.; Mōri, Y.; Yamauchi, T. Chem. Pharm. Bull. 1991, 39, 2709-2711. 